\title{
Preventing Unwanted Pregnancies in Schizophrenia: The Impor- tance of Patient Education
}

\author{
Smita Agarkar $M D^{1,2,3^{*}}$ and Teresitta Velez $B A^{2}$ \\ ${ }^{1}$ Weill Cornell Medicine, New York, USA \\ ${ }^{2}$ Columbia University Vagelos College of Physicians and Surgeons, New York, USA \\ ${ }^{3}$ Gracie Square Hospital, New York, USA
}

*Corresponding author: Smita Agarkar, MD, Weill Cornell Medicine; Adjunct Faculty, Columbia University Vagelos College of Physicians and Surgeons; Psychiatrist, Gracie Square Hospital; New York, USA

The importance of contraception education and family planning is often underestimated by physicians providing care to people with schizophrenia. When compared to the general population, women with schizophrenia tend to have a higher rate of lifetime sexual partners and low utilization of contraception [1,2]. While women with schizophrenia have a comparable number of pregnancies to the general population, these pregnancies are significantly more likely to be unplanned and more importantly, unwanted [1]. In a keystone study done by Miller and Finnerty, 44.3\% of pregnancies among surveyed women with schizophrenia-spectrum disorders were unplanned, but wanted, and $37 \%$ of pregnancies were both unplanned and unwanted [1]. Unplanned and unwanted pregnancies have significant implications for both the health of the patient as well as the fetus.

The most feared risk of an unwanted pregnancy in a person with schizophrenia is the psychotic denial of the pregnancy. Delayed acknowledgment of pregnancy will negatively impact the quality of prenatal care [3]. Sustained denial of the pregnancy can lead to a patient not recognizing signs of labor, ultimately resulting in unaided deliveries [3]. In the postpartum period, people with schizophrenia remain at high risk for acute psychotic exacerbations of their chronic illness, possibly due to low estrogen levels in the immediate postpartum period [4]. Psychotic episodes in the postpartum period are particularly worrisome because they are highly associated with suicide and increased morbidity and mortality [5]. In terms of fetal health, unwanted pregnancy in mothers with psychosis is significantly associated with offspring development of schizophrenia-spectrum disorders in adulthood $[4,6]$.

Current reviews of schizophrenia and sexuality suggest that sexual health discussions outlining contraception options are not occurring regularly [7]. This is not because patients with schizophrenia express discomfort with the topic or refuse to engage, but because healthcare providers fear triggering sexual delusions, view patients with schizophrenia as sexually isolated, feel discomfort discussing these topics, and remain unsure of which member of the care team should be leading these discussions [7]. Notably, it was recently shown that in patients with psychosis, discussions regarding sexual health had no effect on symptom severity [8]. In fact, a review of sexuality and schizophrenia by Kelly and Conley, reported that psychotic symptom severity was actually worsened by patients' lingering concerns of sexual dysfunction [9]. When interviewed, patients with schizophrenia reported feeling prepared to have sexual health discussions and explicitly cite the lack of conversations surrounding intimate relationships as a lapse in their care [9]. This disconnect between provider preconceptions and patient experiences offers insight as to why contraception planning is not currently an integral part of comprehensive care for people with schizophrenia.

Many providers also wrongly assume that patients diagnosed with a serious mental illness, like schizo-

Citation: Agarkar A, Teresitta VBA (2019) Preventing Unwanted Pregnancies in Schizophrenia: The Importance of Patient Education. Int J Womens Health Wellness 5:104. doi.org/10.23937/24741353/1510104 
phrenia, lack decisional-making capacity [10]. A diagnosis of schizophrenia does not inherently signify impaired decision making [10]. Instead, it indicates that a patient's capacity and autonomy may fluctuate over time [11]. Therefore, capacity should be evaluated while a patient is at their baseline and again in the setting of an acute exacerbation of symptoms. While being treated with an optimal antipsychotic regimen, a patient with schizophrenia should be encouraged to engage in discussions regarding their sexual health. These patients can also receive communication skills training and problem-solving strategies that enhance their autonomy [11]. This treatment plan counteracts the aspects of schizophrenia that may impair decisional making capacity, such as command auditory hallucinations, delusional thinking, and disorganized thought [11]. Since patients with schizophrenia may acutely decompensate, providers should consider the use of psychiatric advance directives. Psychiatric advance directives are currently permitted in 25 states and would allow a contraception plan to remain in place should a patient acutely decompensate and have impaired decision making $[12,13]$.

People with schizophrenia should be educated on all contraception options. The utility of barrier protection, such as condoms, must be reinforced routinely in order to prevent the acquisition of sexually transmitted infections [12]. Nevertheless, condoms are thought to be a less than ideal form of contraception for a patient population in which sex is often unexpected and in which impairing substance use is prevalent $[3,12]$. Long-acting injectable contraception (LAls; depot medroxyprogesterone acetate) is commonly thought to have a generally favorable benefit profile while also maximizing ease of use. LAls can be administered at the same time as long-acting injectable antipsychotics without drug-drug interactions, which would promote medication adherence and lessen the hassle of medication administration [3]. LAls also provide protection for 3 months, do not require daily maintenance like oral contraceptive pills (OCPs) which may be difficult in patients with impaired recall, planning, or general medication adherence, and do not require device implantation like IUDs or arm implants which may contribute to delusions of control [3]. While the need to take oral contraceptive pills daily is often viewed as a drawback, the daily regimen may aid some patients' adherence to daily antipsychotic medication [4]. Furthermore, there are growing bodies of evidence suggesting that estrogen may exhibit protective effects against schizophrenic symptom severity [4]. Thus, OCPs could serve a dual purpose of protecting against pregnancy as well as ensuring more stable estrogen levels [4]. OCPs have been shown to increase blood levels of clozapine and chlorpromazine, therefore concurrent use of these medications requires close monitoring of drug levels and side effects [4].
In order to limit the rates of unwanted pregnancies among people with schizophrenia, it is critically important to connect these patients to primary care doctors, gynecologists, or community health coaches who can provide early and regular contraception education. Contraception education should be introduced early in a patient's care because the most frequently endorsed reason for not using birth control is having unplanned/ unexpected sex [3]. Early education would allow people with schizophrenia to make decisions regarding contraception sooner and prepare for the unexpected predicaments that result from increased impulsivity, poorer judgment, and higher rates of substance use often seen with schizophrenia. Thorough education efforts should aim to clarify common misconceptions, such as the idea that drug-induced amenorrhea signifies infertility [4]. Education also needs to be reinforced regularly as studies have shown that while teaching attempts were effective in the short-term, long-term retention of the information required routine reinforcement [12]. Since patients with schizophrenia may have fluctuating or irregular access to the healthcare system, it is important to recognize both regular outpatient appointments and more acute hospital admissions as opportunities to explore sexual health concerns and contraception education.

Patients with schizophrenia often do not receive the same quality of care as that of the general population [14]. This phenomenon is reflected in the fact that U.S. adults with chronic mental illness die 25 years earlier than adults without serious mental illness [14]. Many factors contribute to this reality, including potentially unstable access to care, the lack of coordination between psychiatric and primary care providers, and psycho-socioeconomic factors (e.g. housing instability, low socioeconomic status, lack of social support) [14]. Providers can combat these disparities by reevaluating what it means to provide comprehensive care to patients with schizophrenia. Treatment plans should incorporate family planning and contraception education to ensure that patients with schizophrenia are being cared for in a thorough manner.

\section{References}

1. Miller LJ, Finnerty M (1996) Sexuality, pregnancy, and childrearing among women with schizophrenia-spectrum disorders. Psychiatr Serv 47: 502-506.

2. Miller LJ (1997) Sexuality, reproduction, and family planning in women with schizophrenia. Schizophr Bull 23: 623635.

3. Solari H, Dickson KE, Miller L (2009) Understanding and treating women with schizophrenia during pregnancy and postpartum- Motherisk Update 2008. Can J Clin Pharmacol 16: 22-32.

4. Seeman MV, Ross R (2011) Prescribing contraceptives for women with schizophrenia. J Psychiatr Pract 17: 258-269.

5. Jones I, Chandra PS, Dazzan P, Howard LM (2014) Bipolar disorder, affective psychosis, and schizophrenia in preg- 
nancy and the post-partum period. Lancet 384: 1789-1799.

6. McNeil TF, Schubert EW, Cantor-Graae E, Brossner M, Schubert $P$, et al. (2009) Unwanted pregnancy as a risk factor for offspring schizophrenia-spectrum and affective disorders in adulthood: A prospective high-risk study. Psychol Med 39: 957-965.

7. Seeman MV (2013) Clinical interventions for women with schizophrenia: Pregnancy. Acta Psychiatr Scand 127: 1222.

8. McCann E (2000) The expression of sexuality in people with psychosis: Breaking the taboos. J Adv Nurs 32: 132 138.

9. Kelly DL, Conley RR (2004) Sexuality and schizophrenia: A review. Schizophr Bull 30: 767-779.

10. Ganzini L, Volicer L, Nelson, W, Derse A (2003) Pitfalls in assessment of decision making capacity. Psychosomatics 44: $237-243$

11. McCullough LB, Coverdale JH, Chervenak FA (2002) Ethical challenges of decision making with pregnant patients who have schizophrenia. Am J Obstet Gynecol 187: 696702.

12. Oliver RJ (2013) Contraceptive and sexual health care issues in women with schizophrenia. J Fam Plann Reprod Health Care 39: 289-291.

13. (2019) Psychiatric Advance Directives (PAD). National Alliance on Mental IIIness.

14. Viron M, Baggett T, Hill M, Freudenreich O (2012) Schizophrenia for primary care providers: How to contribute to the care of a vulnerable patient population. Am J Med 125: 223230. 